\title{
Global analysis of the post-SNO solar neutrino data for standard and nonstandard oscillation mechanisms
}

\author{
A. M. Gago, ${ }^{1,2,3}$ M. M. Guzzo, ${ }^{4}$ P. C. de Holanda,${ }^{5}$ H. Nunokawa, ${ }^{4,6}$ O. L. G. Peres, ${ }^{4}$ V. Pleitez,${ }^{6}$ \\ and R. Zukanovich Funchal ${ }^{3}$ \\ ${ }^{1}$ Sección Física, Departamento de Ciencias, Pontificia Universidad Católica del Perú, Apartado 1761, Lima, Peru \\ ${ }^{2}$ Department of Physics, California State University, Dominguez Hills, Carson, California 90747 \\ ${ }^{3}$ Instituto de Física, Universidade de São Paulo, Caixa Postal 66.318, 05315-970 São Paulo SP, Brazil \\ ${ }^{4}$ Instituto de Física Gleb Wataghin, Universidade Estadual de Campinas-UNICAMP, 13083-970 Campinas SP, Brazil \\ ${ }^{5}$ The Abdus Salam International Center for Theoretical Physics, I-34100 Trieste, Italy \\ ${ }^{6}$ Instituto de Física Teórica, Universidade Estadual Paulista, Rua Pamplona 145, 01405-900 São Paulo SP, Brazil
}

(Received 7 December 2001; published 19 March 2002)

\begin{abstract}
What can we learn from solar neutrino observations? Is there any solution to the solar neutrino anomaly which is favored by the present experimental panorama? After SNO results, is it possible to affirm that neutrinos have mass? In order to answer such questions we analyze the current available data from the solar neutrino experiments, including the recent SNO result, in view of many acceptable solutions to the solar neutrino problem based on different conversion mechanisms, for the first time using the same statistical procedure. This allows us to do a direct comparison of the goodness of the fit among different solutions, from which we can discuss and conclude on the current status of each proposed dynamical mechanism. These solutions are based on different assumptions: (a) neutrino mass and mixing, (b) a nonvanishing neutrino magnetic moment, (c) the existence of nonstandard flavor-changing and nonuniversal neutrino interactions, and (d) a tiny violation of the equivalence principle. We investigate the quality of the fit provided by each one of these solutions not only to the total rate measured by all the solar neutrino experiments but also to the recoil electron energy spectrum measured at different zenith angles by the Super-Kamiokande Collaboration. We conclude that several nonstandard neutrino flavor conversion mechanisms provide a very good fit to the experimental data which is comparable with (or even slightly better than) the most famous solution to the solar neutrino anomaly based on the neutrino oscillation induced by mass.
\end{abstract}

DOI: 10.1103/PhysRevD.65.073012

PACS number(s): 14.60.Pq, 13.15.+g, 26.65.+t

\section{INTRODUCTION}

Solar neutrino observations coming from Homestake [1], Kamiokande [2], SAGE [3], GALLEX [4], GNO [5], and Super-Kamiokande (SK) [6] have been suggesting a picture which conflicts with the predictions from the standard solar model (SSM) [7-9], strongly indicating the disappearance of solar electron neutrinos on their way from the Sun to the terrestrial detectors. This has been known for many years as the solar neutrino problem (SNP) [7].

The extraordinary new result from the Sudbury Neutrino Observatory (SNO) [10] inaugurates a new era in the quest for the solution to the long-standing puzzle of missing solar neutrinos. For the first time in the history of solar neutrino observations, a direct indication of the presence of a nonelectron active neutrino component in the solar neutrino flux has been obtained. This cannot be explained by any conceivable modification of the SSM but does require some departure from the standard electroweak theory. The indication of a nonelectron active component is based on the difference of ${ }^{8} \mathrm{~B}$ neutrino flux detected through charged current events in SNO and the neutrino electron elastic scattering events observed by the SK Collaboration, the former obtaining a lower rate than the latter. Such a difference can be explained by the conversion of electron neutrinos into active nonelectron $\left(\nu_{\mu}\right.$ or $\nu_{\tau}$ ) neutrinos along their trajectory from the Sun to the detectors at the Earth $[10,11]$.
As we will see, the hypothesis of no flavor conversion of solar electron neutrinos is strongly in conflict with the prediction of the SSM; it is now only acceptable at a very small confidence level $\left(\sim 10^{-12}\right)$ if only the total rates from solar neutrino experiments are considered (see Sec. IV). The disagreement among the observed solar neutrino data and the theoretical predictions can be relaxed to the $4 \sigma$ level ( 7 $\times 10^{-5}$ ) [12] if one allows all the solar neutrino fluxes to be free parameters in fitting the measured solar neutrino event rates. However, this can only be obtained under the extreme assumption of vanishing ${ }^{7} \mathrm{Be}$ neutrino flux, which is quite difficult to explain.

Several mechanisms can induce neutrino flavor conversion when one assumes that neutrinos are endowed with some properties not present in the minimal standard electroweak theory [13]. The most well known mechanism is the neutrino oscillation induced by mass and mixing [14-17]. The fact that the terrestrial experiments are less sensitive to these resulting nonelectron neutrinos can explain their observed lower counting rates. The purpose of this article is to compare quantitatively the capabilities of several different mechanisms to explain solar neutrino data, for the first time, based on the same statistical procedure.

Readers are invited to take a look at Tables III and IV which summarize our most important results. A number of possible solutions to the solar neutrino anomaly still survives even after SNO results, fitting the data with significant con- 
fidence level. In fact, combined analysis of the data, which includes not only the total rates measured by all solar neutrino experiments but also some information which is independent of the total neutrino flux, namely, the energy spectrum and the zenithal dependence of the data, suggests that the large mixing angle Mikheyev-Smirnov-Wolfenstein (MSW) solution in matter, as well as the mechanisms based on resonant spin-flavor precession, non-standard neutrino interactions and violation of the equivalence principle all provide a fit of the data with the confidence level $\gtrsim 60 \%$.

In Sec. II we briefly review several mechanisms that induce flavor conversion of solar neutrinos, which will be discussed in this work: (a) mass-induced oscillation in vacuum and in matter, (b) resonant spin-flavor precessions induced by a nonvanishing neutrino magnetic moment, (c) the existence of nonstandard neutrino interactions inducing flavorchanging and nonuniversal currents, and (d) the violation of the equivalence principle. In Sec. III the procedure of the statistical analysis used in this work is presented, while our results are given in Sec. IV. Finally, Sec. V is devoted to the discussion of our results.

\section{NEUTRINO CONVERSION MECHANISMS}

The neutrino conversion mechanisms we will consider in this work can be phenomenologically described by the following Schrödingerlike evolution equation:

$$
i \frac{d}{d r}\left[\begin{array}{c}
\nu_{e} \\
\tilde{\nu}
\end{array}\right]=\left[\begin{array}{cc}
A(r) & C(r) \\
C(r) & -A(r)
\end{array}\right]\left[\begin{array}{c}
\nu_{e} \\
\tilde{\nu}
\end{array}\right],
$$

where $r$ is the distance traveled by neutrino, $\nu_{e}$ is the initial electron neutrino state, $\tilde{\nu}$ is the neutrino state to which the conversion mechanism leads and the explicit form of the elements of the $2 \times 2$ Hamiltonian matrix, $A(r)$ and $C(r)$, which in general depend on the position $r$, will be given in the next subsections for each conversion mechanism. For simplicity, we assume neutrino conversion only between two flavors, or more precisely, we assume that neutrino conversion relevant for solar neutrinos can be effectively described, in good approximation, in terms of two flavor conversion.

In all the mechanisms, either in vacuum or in matter, the phenomenon of neutrino mixing occurs and this can be generally expressed as

$$
\left[\begin{array}{c}
\nu_{e} \\
\tilde{\nu}
\end{array}\right]=\left[\begin{array}{cc}
\cos \widetilde{\theta} & \sin \widetilde{\theta} \\
-\sin \widetilde{\theta} & \cos \widetilde{\theta}
\end{array}\right]\left[\begin{array}{l}
\nu_{1} \\
\nu_{2}
\end{array}\right],
$$

where $\widetilde{\theta}$ is the mixing angle which relates the neutrino flavor and the propagating eigenstates $\nu_{1}$ and $\nu_{2}$ in matter or in vacuum. Note that $\widetilde{\theta}$ can be defined even for massless neutrinos as in the case of NSNI or VEP.

For all the mechanisms we analyze in this work, we do not consider the case where $\tilde{\nu}$ is a sterile (or electroweak singlet) neutrino [18] because of the present indication of the presence of nonelectron active component in the solar neutrino flux, which is provided by the combined information from SNO and SK results. Namely, neutrino conversion of solar $\nu_{e}$ only into a sterile neutrino is not favored by the current data.

\section{A. Mass-induced oscillation (MIO)}

Let us first consider the most popular conversion mechanism, the one induced by neutrino mass and mixing. If one assumes that neutrinos are massive, the flavor eigenstates do not coincide, in general, with the mass eigenstates, leading to neutrino flavor oscillation in vacuum $[14,15]$ as well as in matter where it can be resonantly enhanced $[16,17]$. The latter is known as the Mikheyev-Smirnov-Wolfenstein (MSW) effect. It is generally believed that this is the most plausible mechanism which can induce neutrino oscillation since to introduce mass and mixing in the leptonic sector is the simplest and most natural extensions to the standard electroweak model, so theoretically well motivated.

In both scenarios, MIO can convert solar electron neutrinos into neutrinos of a different flavor and consequently explain the deficit of an observed solar neutrino with respect to the predictions of the SSM. We will refer to these type of solutions as standard solutions to the SNP; they are very well described in recent Refs. [19,20]. MIO requires in Eq. (1) that

$$
\begin{aligned}
& A(r)=-\frac{\delta m^{2}}{4 E} \cos 2 \theta+\frac{G_{F}}{\sqrt{2}} N_{e}(r), \\
& C(r)=\frac{\delta m^{2}}{4 E} \sin 2 \theta,
\end{aligned}
$$

where $\delta m^{2} \equiv m_{2}^{2}-m_{1}^{2}$ is the mass squared difference of the two neutrinos involved, $\theta$ is the vacuum mixing angle, $G_{F}$ is the Fermi constant, $E$ is the neutrino energy, and $N_{e}(r)$ is the electron number density at position $r$, and here $\tilde{\nu}$ is identified with $\nu_{\mu}$ or $\nu_{\tau}$ (or their linear combination). We compute the conversion probability using the analytic formulas, properly taking into account the neutrino production distributions, as well as the Earth matter effect as in Ref. [21]. The relevant oscillation parameters, which must be determined by the fit to experimental data, are $\delta m^{2}$ and $\theta$. Recent post-SNO analyses can be found in Ref. [22]. Depending on the allowed parameter values, the solutions based on this mechanism are classified into large mixing angle (LMA) MSW, small mixing angle (SMA) MSW, low- $\delta m^{2}$ (LOW) MSW, and vacuum oscillation (VAC) solutions.

\section{B. Resonant spin-flavor precession (RSFP)}

Assuming neutrinos have a nonvanishing transition magnetic moment, electron neutrinos interacting with the solar magnetic field can be spin-flavor converted into active nonelectron antineutrinos [23], if they are of Majorana type. Here we do not consider the case of Dirac-type neutrinos since it involves a sterile one. Furthermore, such spin-flavor precession of neutrinos can be resonantly enhanced in matter [24], in close analogy to the MSW effect [16,17]. RSFP could strongly depend on the neutrino energy and provoke 
different suppressions for each portion of the solar neutrino energy spectrum, in a similar way as in the case of the MSW effect. In fact it has been known that RSFP provides a satisfactory description [25-27] of the actual experimental panorama [1-6]. However, see a recent work [28] where it was found that the nonresonant spin-flavor precession can also explain the solar neutrino data.

The time evolution of neutrinos interacting with a magnetic field $B$ through a nonvanishing neutrino magnetic moment $\mu_{\nu}$ in matter is governed by Eq. (1) by defining $A(r)$ and $C(r)$ as [24]

$$
\begin{aligned}
& A(r)=-\frac{\delta m^{2}}{4 E}+\frac{1}{\sqrt{2}} G_{F}\left[N_{e}(r)-N_{n}(r)\right], \\
& C(r)=\mu_{\nu} B(r),
\end{aligned}
$$

where $N_{n}(r)$ is the neutron number densities, $\mu_{\nu}$ is the transition magnetic moment between the two neutrino involved, $B(r)$ is the solar magnetic field, and here $\tilde{\nu}$ is identified with $\bar{\nu}_{\mu}$ or $\bar{\nu}_{\tau}$ (or their combination). We assume, for simplicity, that the mixing angle is zero in this mechanism. Note that, roughly speaking, $\mu_{\nu} B(r)$ is playing the same role as the mixing term $\left(\delta m^{2} / 4 E\right) \sin 2 \theta$, which appeared in the standard mass-induced oscillation mechanism. In this work we assume, as a reference value, the magnitude of the neutrino magnetic moment to be $\mu_{\nu}=10^{-11} \mu_{B}$ ( $\mu_{B}$ is the Bohr magneton). Since the relevant quantity is only the product of the neutrino magnetic moment and the magnetic field, it must be understood that if $\mu_{\nu}$ is taken to be smaller, the solar magnetic field must be properly increased to achieve the same effect.

The RSFP mechanism crucially depends on the solar magnetic field profile along the neutrino trajectory. In our present analysis we assume a particular solar magnetic field profile which could explain well the solar neutrino data (before the SNO result) with a relatively weak magnetic field $(\sim 10 \mathrm{kG})$, which was considered in a number of works in Refs. [26,27]. The profile we will use has a triangular configuration in the solar convective zone. For definiteness, we take the one which is found in Ref. [29]. The profile has a vanishing magnetic field in the internal part of the Sun, linearly growing from $r=0.7$ to $r=0.85$, where it achieves its maximum value $B_{\max }$ and begins to linearly decline until the surface of the Sun, where $r=1$. Here $r$ is the radial distance from the center of the Sun normalized by the solar radius. In this work, we consider $B_{\max }$ up to $500 \mathrm{kG}$ taking into consideration the upper limit $(300 \mathrm{kG})$ of the magnetic field at the bottom of the convective zone obtained in Ref. [30]. Once the shape of the magnetic field profile is fixed, the relevant parameters which must be determined by the fit can be chosen to be $\delta m^{2}$ and $B_{\max }$.

We compute the conversion probability by numerically integrating the evolution equation as in Ref. [27]. For simplicity, we assumed that all the neutrinos are created in the solar center and we neglected the Earth matter effect, as discussed in Ref. [27].

\section{Nonstandard neutrino interactions (NSNI)}

In his seminal paper Wolfenstein [16] observed that NSNI with matter can also generate neutrino oscillation even without flavor mixing in vacuum. Some explicit examples of such NSNI-induced neutrino oscillation were considered in Refs. [31,32]. It has been shown that NSNI could be relevant to solar neutrinos propagating in the solar matter along their path from the core of the Sun to its surface [31-33] as well as in the Earth until they reach the detector.

The evolution equations for massless neutrinos (or neutrinos with degenerate mass) having such NSNI in matter can be phenomenologically expressed by Eq. (1) with the following definitions of $A(r)$ and $C(r)[31,32]$ :

$$
\begin{aligned}
& A(r)=\frac{1}{\sqrt{2}} G_{F}\left[N_{e}(r)-\epsilon_{f}^{\prime} N_{f}(r)\right], \\
& C(r)=\sqrt{2} G_{F} \epsilon_{f} N_{f}(r),
\end{aligned}
$$

where $\epsilon_{f}^{\prime}$ and $\epsilon_{f}$ are the phenomenological parameters which characterize the strength of the NSNI with fermion $f$ whose number density is given by $N_{f}(r)$ with $f=u$ or $d$ quark, and here $\tilde{\nu}$ is identified with $\nu_{\mu}$ or $\nu_{\tau}$ (or their combination).

Here, by taking into account the charge neutrality, $N_{f}$ can be written in terms of electron and neutron number densities, as follows:

$$
N_{f}(r)= \begin{cases}N_{n}(r)+2 N_{e}(r) & (f=u), \\ 2 N_{n}(r)+N_{e}(r) & (f=d) .\end{cases}
$$

The parameters $\epsilon_{f}$ appearing in the off-diagonal elements of the Hamiltonian matrix are responsible for flavor changing neutrino interactions; it plays a similar role to the mixing term $\left(\delta m^{2} / 4 E\right) \sin 2 \theta$ in the MIO mechanism. It exists even if there is no neutrino mixing in vacuum. On the other hand, $\epsilon_{f}^{\prime}$ appearing in the diagonal element, which is responsible for flavor diagonal neutrino interactions with matter [31] somehow plays a role similar to the mass squared difference in the MIO mechanism, since this term leads to resonantly enhanced conversion when its magnitude coincides with that of the standard electroweak neutrino interactions at some point $r_{\text {res }}$ along the neutrino trajectory, satisfying the resonance condition,

$$
\epsilon_{f}^{\prime} N_{f}\left(r_{\text {res }}\right)=N_{e}\left(r_{\text {res }}\right) .
$$

An immediate consequence is that if $f$ was to be identified with electron, it cannot induce resonant neutrino flavor conversion [34].

An important characteristic of this mechanism is that the conversion probability does not depend on neutrino energy as it is understood from the evolution equation. Nevertheless this mechanism can explain quite well the solar neutrino data which imply strong energy-dependent conversion. The reason is that even though the conversion probability itself is completely energy independent, after taking an average of the probability over production distributions, different neutrinos from different nuclear reactions in the Sun $\left(p p,{ }^{8} \mathrm{~B}\right.$, 
TABLE I. Observed solar neutrino data used in this analysis presented together with the theoretical predictions of "BP2000 $\mathrm{SSM}+\mathrm{New}{ }^{8} \mathrm{~B} "[8,9]$.

\begin{tabular}{|c|c|c|c|}
\hline Experiments & $\begin{array}{l}\text { Observed } \\
\text { rates }\end{array}$ & $\begin{array}{c}\text { SSM } \\
\text { predictions }\end{array}$ & Units \\
\hline Homestake & $2.56 \pm 0.23$ & $8.59_{-1.2}^{+1.1}$ & SNU \\
\hline SAGE & $77.0 \pm 6 . \pm 3$ & $130_{-7}^{+9}$ & SNU \\
\hline $\begin{array}{c}\text { GALLEX } \\
+ \text { GNO }\end{array}$ & $73.9 \pm 4.7 \pm 4.0$ & $130_{-7}^{+9}$ & SNU \\
\hline SK & $0.391 \pm 0.014$ & $1.00_{-0.15}^{+0.14}$ & $5.93 \times 10^{6} \mathrm{~cm}^{-2} \mathrm{~s}^{-1}$ \\
\hline SNO CC & $0.296 \pm 0.024$ & $1.00_{-0.15}^{+0.14}$ & $5.93 \times 10^{6} \mathrm{~cm}^{-2} \mathrm{~s}^{-1}$ \\
\hline
\end{tabular}

${ }^{7} \mathrm{Be}$, etc.) can have different final average probability due to the fact that their production distributions are different, provided that resonant conversion occurs close to the solar core ( $\sim 10 \%$ or so of the solar radius).

Following Ref. [33], we first compute the conversion probability using the analytic formulas for a given production point in the Sun and then take the average over the production distribution for each neutrino source. We also take into account the Earth regeneration effect, which is important for some region of the parameter space.

As it was observed in Ref. [31], only the $\nu_{e} \rightarrow \nu_{\tau}$ conversion is compatible with the existing phenomenological constraints on $\epsilon_{f}$ and $\epsilon_{f}^{\prime}$. In this way, the relevant parameters which must be determined by the fit for this mechanism are $\epsilon_{f}^{\prime}$ and $\epsilon_{f}$.

\section{Violation of the equivalence principle (VEP)}

It has been proposed that violation of the equivalence principle could induce neutrino flavor oscillation even if neutrinos are massless $[35,36]$. In this scenario, neutrino mixing and flavor oscillation can be induced if two (or more) neutrinos involved have different gravitational couplings which imply VEP. In this case, weak interacting eigenstates and gravitational interacting eigenstates can be different and will be related by a unitary transformation that can be parametrized, assuming only two neutrino flavors, by a single parameter, the mixing angle $\theta_{G}$ similar to the case of neutrino mixing in vacuum induced by mass.

The evolution equations for these flavors, which are assumed to be degenerate in mass, propagating through the gravitational potential $\phi(r)$ in the absence of matter is given by Eq. (1) with $[35,36]$ :

$$
\begin{aligned}
& A(r)=2 E \phi(r) \delta \gamma \cos 2 \theta_{G}, \\
& C(r)=2 E \phi(r) \delta \gamma \sin 2 \theta_{G},
\end{aligned}
$$

where $\delta \gamma$ is the quantity which measures the magnitude of the violation of the equivalence principle, the difference of the gravitational couplings between the two neutrinos involved normalized by the sum. Here $2 E \phi(r) \delta \gamma \sin 2 \theta_{G}$ plays a similar role to the mixing term $\left(\delta m^{2} / 4 E\right) \sin 2 \theta$ in the MIO mechanism; this means the VEP mechanism gives rise to an oscillation length inversely proportional to the neutrino energy $E$, while MIO expects it to be directly proportional to E.

As in Ref. [37], we take $\phi(r)$ to be constant $\left(\sim 10^{-5}\right)$, assuming that the local supercluster contribution is the dominant one [38]. The relevant parameters which must be determined by the fit in this mechanism are $|\phi \delta \gamma|$ and $\theta_{G}$. We consider the product of $\phi$ and $\delta \gamma$ since the former has a large uncertainty and only the product is relevant in the fit. Similar to the mass-induced oscillation, we can have two types for the VEP mechanism: (a) VEP-induced MSW-like resonant conversion and (b) VEP vacuum conversion. The former was first discussed in Ref. [36], and then analyzed by several authors [39]. However, Ref. [40] showed that it is not a good solution because the required magnitude of the parameters is incompatible with the CCFR experiment results [41] which exclude $|\phi \delta \gamma|$ larger than $\sim 10^{-23}$. For the latter one, it has been shown that this mechanism is a new solution for the solar neutrino anomaly [37]. Recent analysis of the VEP vacuum solution was discussed in Ref. [42].

We calculate the conversion probability using the analytic formulas as in Ref. [37]. Similar to the case of vacuum oscillation solution in the MIO scenario, we can neglect, as good approximations, the neutrino production distributions and the Earth matter effect [37].

\section{STATISTICAL PROCEDURE}

Our main goal is to determine the allowed values of the relevant parameters for each one of the mechanisms that can explain the experimental observations without modifying the SSM predictions and to evaluate the quality of the fit. In order to achieve our goal, we adopt the minimum $\chi^{2}$ statistical treatment of the data following the description found in Ref. [43] which was also employed in Refs. $[21,20,27,33,37,44]$ but with some modifications.

In this work, we perform three kinds of analysis using three $\chi^{2}$ functions, $\chi_{R}^{2}$ for the analysis of the rates only, $\chi_{\mathrm{fi}}^{2}$ for the flux-independent analysis, and $\chi_{\mathrm{comb}}^{2} \equiv \chi_{R}^{2}+\chi_{\mathrm{fi}}^{2}$ for the combined analysis, where the definitions of $\chi_{R}^{2}$ and $\chi_{\mathrm{fi}}^{2}$ are given in the following subsections. For each case, we minimize the $\chi^{2}$ function in order to determine the best fitted parameters as well as its minimum value $\chi_{\min }^{2}$, which is relevant for the evaluation of the goodness of fit. The allowed parameter region can be determined by the condition

$$
\chi^{2}<\chi_{\min }^{2}+\Delta \chi^{2}
$$

where $\Delta \chi^{2}=4.61,5.99$, and 9.21 for $90 \%, 95 \%$, and $99 \%$ confidence levels (C.L.), respectively.

\section{A. $\chi^{2}$ for the analysis of the rates}

First we describe the $\chi^{2}$ for the analysis of the total event rate measured by the chlorine $(\mathrm{Cl})$ experiment [1], the gallium detectors GALLEX and GNO $[4,5]$ (we use the combined results of GALLEX and GNO) and SAGE [3], the water Cherenkov experiment SK [6] and also the recent neutrino-deuterium charged current data by SNO [10]. For 
TABLE II. Observed SK zenith-spectrum event rates in units of the BP2000 [8], taken from Table I of Ref. [47]. Errors are only statistical. For the energy-uncorrelated and energy-correlated systematic uncertainties, see Table I in Ref. [47].

\begin{tabular}{lccccccc}
\hline \hline & & \multicolumn{7}{c}{ Observed rates and statistical errors in units of SSM } \\
& Day & Mantle 1 & Mantle 2 & Mantle 3 & Mantle 4 & Mantle 5 & Core \\
$\cos \theta_{z}$ & $-1.00-0.00$ & $0.00-0.16$ & $0.16-0.33$ & $0.33-0.50$ & $0.50-0.67$ & $0.67-0.84$ & $0.84-1.00$ \\
$E_{e}(\mathrm{MeV})$ & & & & & & \\
\hline $5.0-5.5$ & & & & $0.436 \pm 0.046$ & & \\
$5.5-6.5$ & $0.431 \pm 0.022$ & $0.464 \pm 0.060$ & $0.410 \pm 0.055$ & $0.442 \pm 0.048$ & $0.453 \pm 0.048$ & $0.495 \pm 0.054$ \\
$6.5-8.0$ & $0.461 \pm 0.013$ & $0.524 \pm 0.036$ & $0.506 \pm 0.033$ & $0.438 \pm 0.028$ & $0.466 \pm 0.027$ & $0.424 \pm 0.030$ & $0.409 \pm 0.033$ \\
$8.0-9.5$ & $0.437 \pm 0.014$ & $0.449 \pm 0.038$ & $0.482 \pm 0.036$ & $0.460 \pm 0.031$ & $0.503 \pm 0.031$ & $0.461 \pm 0.034$ & $0.439 \pm 0.037$ \\
$9.5-11.5$ & $0.434 \pm 0.015$ & $0.432 \pm 0.042$ & $0.493 \pm 0.040$ & $0.446 \pm 0.034$ & $0.448 \pm 0.034$ & $0.435 \pm 0.037$ & $0.484 \pm 0.044$ \\
$11.5-13.5$ & $0.456 \pm 0.026$ & $0.496 \pm 0.071$ & $0.290 \pm 0.055$ & $0.394 \pm 0.053$ & $0.477 \pm 0.056$ & $0.439 \pm 0.061$ & $0.465 \pm 0.068$ \\
$13.5-16.0$ & $0.482 \pm 0.056$ & $0.532 \pm 0.155$ & $0.775 \pm 0.171$ & $0.685 \pm 0.141$ & $0.607 \pm 0.130$ & $0.471 \pm 0.128$ & $0.539 \pm 0.153$ \\
$16.0-20.0$ & & & & $0.476 \pm 0.149$ & & \\
\hline \hline
\end{tabular}

simplicity we do not consider the result from the Kamiokande experiment [2] as it is consistent with the current SK one and has much larger experimental errors. All the solar neutrino data for the analysis of the rates used in this work are summarized in Table I. In all our calculations of the theoretical predictions we have used the latest "BP2000 $\mathrm{SSM}+\mathrm{New}{ }^{8} \mathrm{~B}$ " $[8,9]$ fluxes, which include the recent new measurement of $S_{17}(0)$ [45]. Our $\chi^{2}$ function is defined as follows:

$$
\chi_{R}^{2}=\sum_{i, j=1, \ldots, 5}\left[R_{i}^{\mathrm{th}}-R_{i}^{\mathrm{obs}}\right]\left[\sigma_{R}^{2}\right]_{i j}^{-1}\left[R_{j}^{\mathrm{th}}-R_{j}^{\mathrm{obs}}\right]
$$

where $R_{i}^{\text {th }}$ and $R_{i}^{\text {obs }}$ denote, respectively, the predicted and the measured value for the event rates of the five solar experiments considered, $i=\mathrm{Cl}$, GALLEX/GNO, SAGE, SK, SNO.

In order to compute the predictions for the rates, for chlorine, gallium and SK detectors, we follow Refs. [21,20]. For SNO, we compute the rates using the neutrino charged current cross section on deuterium, $\nu_{e}+d \rightarrow p+p+e^{-}$, as given in Ref. [46] and taking into account the energy resolution as described in Ref. [10]. The error matrix $\sigma_{R}$ contains both experimental (systematic and statistical) and theoretical errors [43].

\section{B. $\chi^{2}$ for flux-independent analysis: SK zenith and spectrum}

Our final result is the fit derived from the combined analysis of all presently available solar neutrino data, which includes the flux-independent information presented by the SK Collaboration [47]. We include this information using the SK data split simultaneously on seven zenith bins and eight spectrum bins. We will refer to these as the SK zenithspectrum data. These data are summarized in Table II. For the first and last spectrum bins the zenith data are grouped together in one data point due to low detection rate at these bins; in total 44 are data points related to flux-independent information. We used the statistical and systematic energycorrelated and uncorrelated uncertainties listed in Ref. [47]. The systematic uncertainties are assumed to be fully correlated in the zenith angle splitting. We then used the following $\chi^{2}$ expression for the flux-independent information:

$$
\chi_{\mathrm{fi}}^{2}=\sum_{i=1,44}\left[\alpha R_{\mathrm{SK}, i}^{\mathrm{th}}-R_{\mathrm{SK}, i}^{\mathrm{obs}}\right]\left[\sigma_{\mathrm{fi}}(i, j)^{2}\right]^{-1}\left[\alpha R_{\mathrm{SK}, i}^{\mathrm{th}}-R_{\mathrm{SK}, i}^{\mathrm{obs}}\right],
$$

where $R_{\mathrm{SK}, i}^{\mathrm{th}}$ is the theoretically expected event rates for the $i$ th bin computed by using the ${ }^{8} \mathrm{~B}$ neutrino energy spectrum given in Ref. [48] normalized to the BP2000 SSM value [8], $R_{\mathrm{SK}, i}^{\mathrm{obs}}$ is the corresponding observed rate reported by the SK

TABLE III. Comparison of the existing solutions to the SNP when only the total rates are taken into consideration in the statistical analysis. For each one of the indicated mechanisms the best fit values of the relevant parameters are shown, followed by the corresponding $\chi_{\min }^{2}$ and its confidence level. In the first row we see the result of the poor fit of the SSM predictions to the data. All other solutions present two free parameters to fit five experimental data points, resulting in 3 d.o.f.

\begin{tabular}{|c|c|c|c|c|}
\hline MIO & $\delta m^{2}\left(\mathrm{eV}^{2}\right)$ & $\tan ^{2} \theta$ & & \\
\hline Vacuum & $6.80 \times 10^{-11}$ & 0.425 & 3.02 & $39 \%$ \\
\hline SMA & $7.91 \times 10^{-6}$ & $1.59 \times 10^{-3}$ & 4.60 & $20 \%$ \\
\hline LMA & $2.80 \times 10^{-5}$ & 0.320 & 2.62 & $45 \%$ \\
\hline LOW & $1.05 \times 10^{-7}$ & 0.743 & 10.5 & $1.5 \%$ \\
\hline \multirow[t]{2}{*}{ RSFP } & $\delta m^{2}\left(\mathrm{eV}^{2}\right)$ & $B_{\max }(\mathrm{kG})$ & & \\
\hline & $1.11 \times 10^{-8}$ & 338 & 1.01 & $80 \%$ \\
\hline NSNI & $\epsilon^{\prime}$ & $\epsilon$ & & \\
\hline$d$ quarks & 0.599 & $3.22 \times 10^{-3}$ & 0.72 & $87 \%$ \\
\hline$u$ quarks & 0.428 & $1.39 \times 10^{-3}$ & 0.73 & $87 \%$ \\
\hline \multirow[t]{2}{*}{ VEP } & $|\phi \Delta \gamma|$ & $\sin ^{2} 2 \theta_{G}$ & & \\
\hline & $1.56 \times 10^{-24}$ & 1.0 & 6.23 & $10 \%$ \\
\hline
\end{tabular}

\begin{tabular}{lcc}
\hline \hline Mechanism & $\chi_{\min }^{2}$ & C.L. \\
SSM with & 62.5 & $4 \times 10^{-12}$ \\
no oscillation & $(5$ d.o.f. $)$ &
\end{tabular}


TABLE IV. Same as in Table III but for the statistical analysis, taking into consideration not only the total rates but also the energy spectrum and zenithal dependence of the data.

\begin{tabular}{|c|c|c|c|c|}
\hline Mechanism & & & $\chi_{\min }^{2}$ & C.L. \\
\hline $\begin{array}{l}\text { SSM with } \\
\text { no oscillation }\end{array}$ & & & $\begin{array}{c}100.0 \\
\text { (48 d.o.f.) }\end{array}$ & $1.6 \times 10^{-5}$ \\
\hline MIO & $\delta m^{2}\left(\mathrm{eV}^{2}\right)$ & $\tan ^{2} \theta$ & & \\
\hline Vacuum & $4.65 \times 10^{-10}$ & 1.89 & 46.1 & $47 \%$ \\
\hline SMA & $4.93 \times 10^{-6}$ & $4.35 \times 10^{-4}$ & 61.5 & $6.3 \%$ \\
\hline LMA & $6.15 \times 10^{-5}$ & 0.349 & 38.7 & $75 \%$ \\
\hline LOW & $1.01 \times 10^{-7}$ & 0.783 & 45.0 & $38 \%$ \\
\hline \multirow[t]{2}{*}{ RSFP } & $\delta m^{2}\left(\mathrm{eV}^{2}\right)$ & $B_{\max }(\mathrm{kG})$ & & \\
\hline & $1.22 \times 10^{-8}$ & 440 & 38.4 & $78 \%$ \\
\hline NSNI & $\epsilon^{\prime}$ & $\epsilon$ & & \\
\hline$d$ quarks & 0.599 & $3.23 \times 10^{-3}$ & 37.9 & $80 \%$ \\
\hline$u$ quarks & 0.428 & $1.40 \times 10^{-3}$ & 37.9 & $80 \%$ \\
\hline \multirow[t]{2}{*}{ VEP } & $|\phi \Delta \gamma|$ & $\sin ^{2} 2 \theta_{G}$ & & \\
\hline & $1.59 \times 10^{-24}$ & 1.0 & 42.9 & $60 \%$ \\
\hline
\end{tabular}

Collaboration [47], $\alpha$ is a free parameter to avoid doublecounting of the SK total rate in the statistical treatment, and $\sigma_{\mathrm{fi}}(i, j)$ is the $44 \times 44$ error matrix for SK zenith-spectrum data. This treatment is slightly different from the one presented in recent solar neutrino analysis $[9,11,22]$ which used the SK elastic scattering spectrum data taken during the day and the night separately whereas we used the SK spectrum measured at different zenith angle (night spectrum data are divided into six zenith bins).

\section{RESULTS}

In this section we discuss the results of our statistical analysis. In Table III a comparison of the existing solutions to the SNP, when only the total rates are taken into consideration in the statistical analysis, is presented. For each one of the indicated mechanisms the best fit values of the relevant parameters are shown, followed by the corresponding $\chi_{\min }^{2}$ and its confidence level. In the first row one can find the result of the poor fit of the SSM predictions to the data: $\chi_{\min }^{2}=62.5$ for 5 degrees of freedom (d.o.f.) which represents a confidence level of only $\sim 10^{-12}$. All the other solutions present two free parameters to fit five experimental data points, resulting in 3 d.o.f. The largest confidence level (87\%), shown in Table III, of the fit to the observed data is achieved by the solutions based on neutrino NSNI and the second largest one (80\%) is achieved by the RSFP solution. We note that the RSFP solution has an extra freedom in choosing the solar magnetic field profile. Indeed we found that the RSFP solution is rather sensitive to the relatively
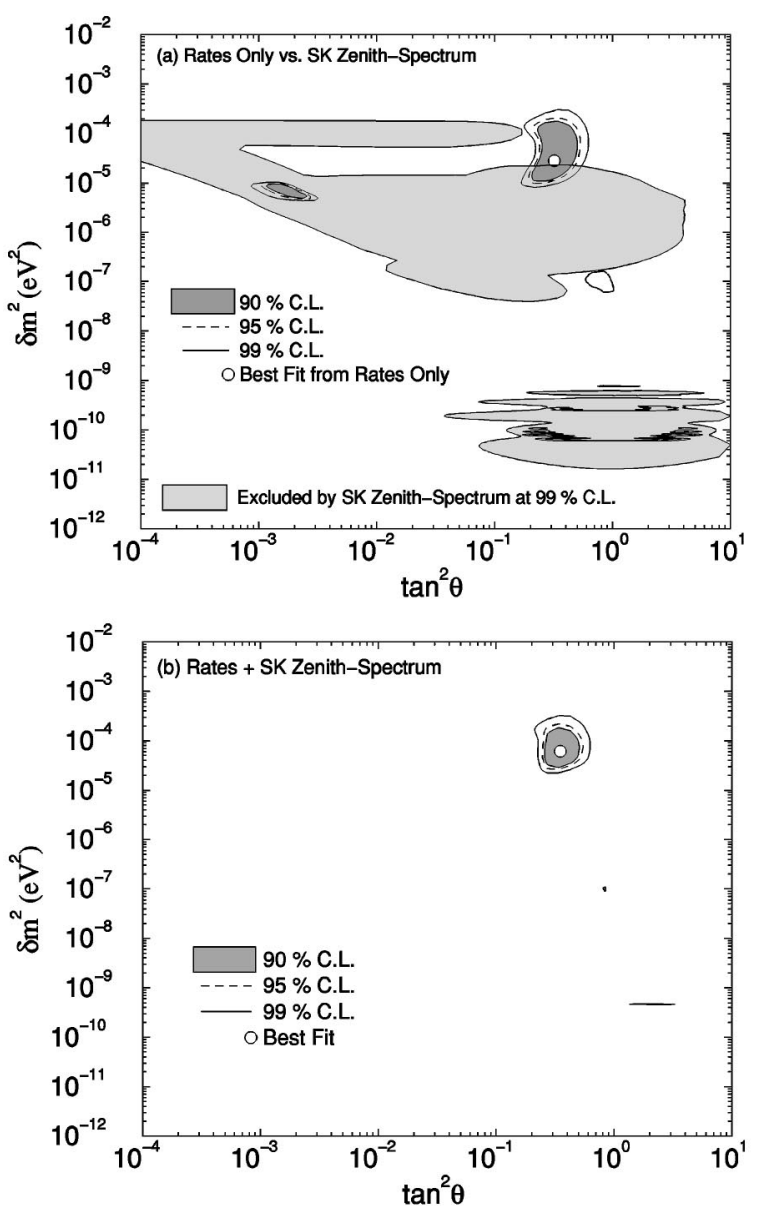

FIG. 1. In (a) the allowed region for the MIO solutions to the SNP at $90 \%, 95 \%$, and 99\% C.L. with rates only as well as the excluded region at $99 \%$ C.L. from the SK zenith-spectrum information are shown. In (b) the allowed region from the combined information from rates and zenith-spectrum data is presented.

small change of the magnetic field profile. We found that if we use the similar but different magnetic field profile used in Ref. [27], which has the peak at $r=0.65$, somewhat inner region of the Sun, solutions exist only for $B_{\max }$ larger than $\sim 100 \mathrm{kG}$. Among the solutions with no such extra freedom, $\mathrm{MIO}$ in vacuum is the second best one $(45 \%)$.

For the no-oscillation hypotheses, in the case where only the SK zenith-spectrum information is used, we obtain $\chi_{\mathrm{fi}}^{2}$ $=37.7$ with $\alpha=0.446$, for 43 d.o.f., which is compatible with the experimental data at $70 \%$ C.L. These results imply that there are no strong distortions in the the shape of SK zenith spectra when compared to the SSM predictions, and the observed data are consistent with the no-oscillation hypothesis provided that the overall rate is normalized.

When we include in the statistical analysis not only the total rates but also the energy spectrum and zenithal dependence of the data coming from the SK experiment, then the panorama changes as summarized in Table IV. As in Table III, the first row shows the fit of the SSM predictions to the combined data: $\chi_{\min }^{2}=100.0$ for 48 d.o.f., which represents a confidence level of $\sim 10^{-5}$. The solutions listed in Table IV present two free parameters to fit 48 experimental data 

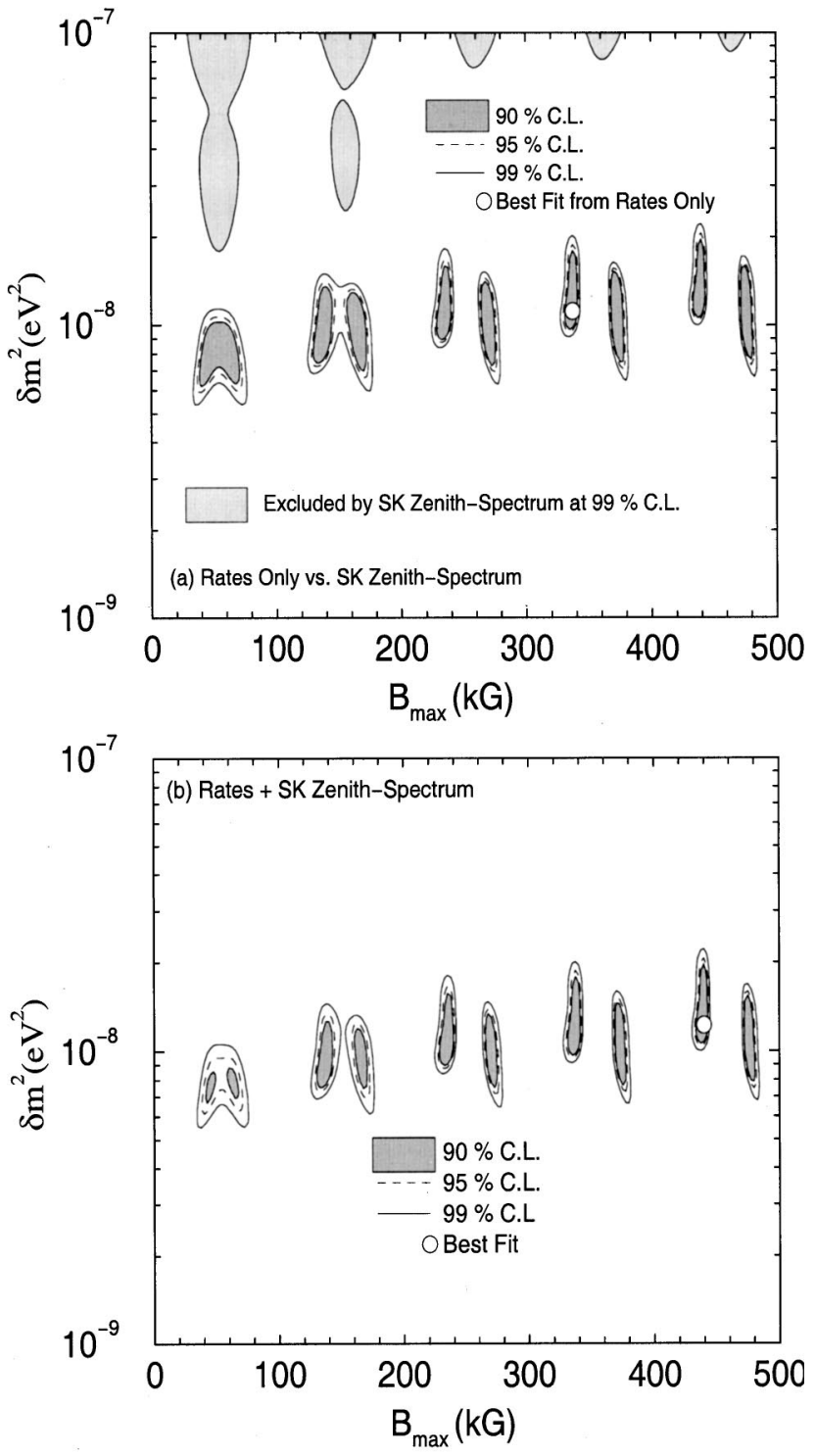

FIG. 2. Same as Fig. 1 but for the RSFP solution in the $B_{\text {max }}-\delta m^{2}$ plane.

points, resulting in 46 d.o.f. Here, the large mixing angle MSW solution based on MIO, as well as RSFP and NSNI solutions are equally probable all having $\gtrsim 80 \%$ C.L. The VEP solution also provides a very good fit, $60 \%$ C.L.

In Figs. 1(a)-5(a) we present for all the conversion mechanisms (i) the allowed parameter region determined from the total rates only and (ii) the excluded parameter region determined only from the SK zenith-spectrum information. In Figs. 1(b)-5(b) we present the allowed parameter region determined from the combined data of the rates and the SK zenith-spectrum information.

It is worthwhile to note that for the MIO and NSNI cases, Figs. 1(a), 3(a), and 4(a), in some parameter regions, there is a significant overlap between the region allowed by rates only and the one excluded by the SK zenith-spectrum information. Namely, there is a strong conflict between the rates and the SK zenith-spectrum fit for some parameters and this
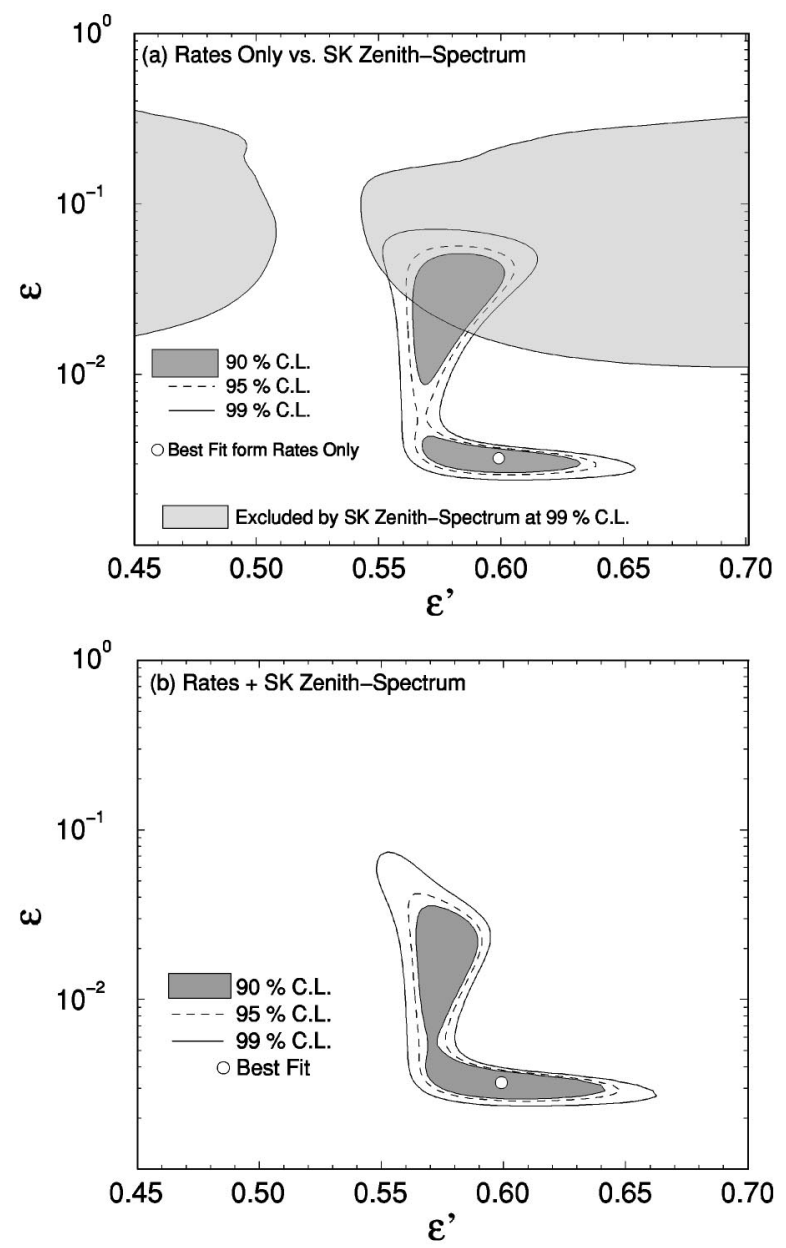

FIG. 3. Same as Fig. 1 but for the solution based on NSNI with $d$ quarks in the parameter space of $\varepsilon\left(\equiv \epsilon_{d}\right)$ and $\varepsilon^{\prime}\left(\equiv \epsilon_{d}^{\prime}\right)$.

is the reason why the allowed region decreases significantly when both data are combined as we can see in Figs. 1(b), 3(b), and 4(b). On the other hand, for the RSFP and VEP cases, there is no such kind of strong conflict between rates and and SK zenith-spectrum fit and therefore, the allowed region for the combined data is rather similar to the one determined only by the rates as we can see in Figs. 2 and 5.

\section{DISCUSSIONS}

Even though the conventional mass-induced oscillation mechanism, which is theoretically well motivated, can be considered as the most plausible solution to the solar neutrino problem, it is important to realize that solutions based on new physics in the neutrino sector, such as a large neutrino magnetic moment, neutrino flavor-changing and nonuniversal processes, in violation of the equivalence principle, can still be viable solutions that provide a fit to the solar neutrino data, which are comparable to the solution based on the conventional mass-induced neutrino oscillation, as we showed in this work.

We also note that some of these mechanisms which do not require neutrino mass in the fit to the solar neutrino data do 

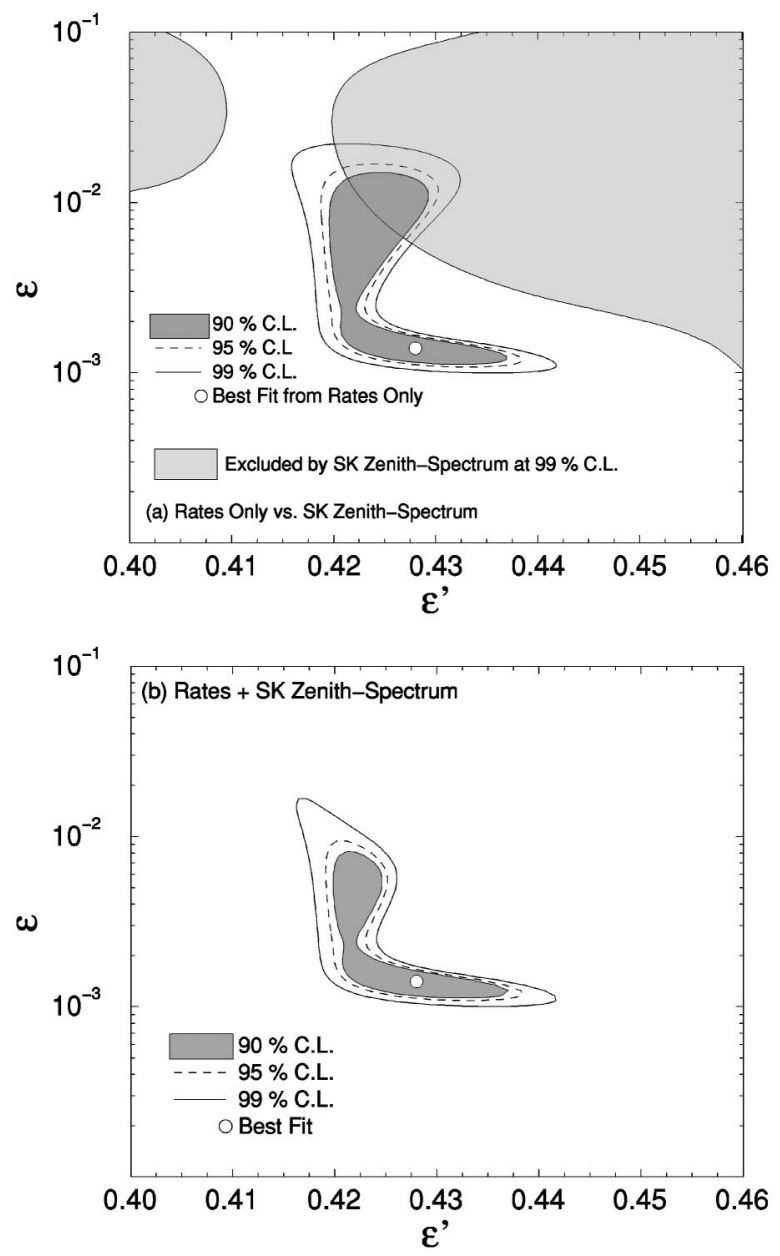

FIG. 4. Same as in Fig. 3 but for NSNI with $u$ quarks in the parameter space of $\varepsilon\left(\equiv \epsilon_{u}\right)$ and $\varepsilon^{\prime}\left(\equiv \epsilon_{u}^{\prime}\right)$.

have some close relation with neutrino mass generation. For example, in our phenomenological approach, we ignored neutrino mass in the solution based on nonstandard neutrino interactions but there is no available model that prevents neutrinos from acquiring mass at a radiative level, if flavor changing and flavor nonuniversal interactions with quarks are present, even if no tree-level mass terms appear in the model.

It is fundamental to mention that it is difficult to explain the atmospheric neutrino problem [49] as well as the LSND anomalies [50] by these alternative mechanisms [51]. Furthermore, while mass-induced oscillation and resonant spinflavor precession solutions to the SNP can be easily conciliated with the standard neutrino oscillation solution to the atmospheric neutrino problem, it is not a trivial task to answer if the nonstandard flavor-changing and nonuniversal neutrino interactions and violation of equivalence principle solutions to the SNP modify or even damage this standard solution to the atmospheric neutrino anomaly [52].

Having this picture in mind we conclude that no specific solution is preferred by the current solar neutrino data, although some solutions may have difficulties in reconciling atmospheric neutrino observations. We emphasize that the
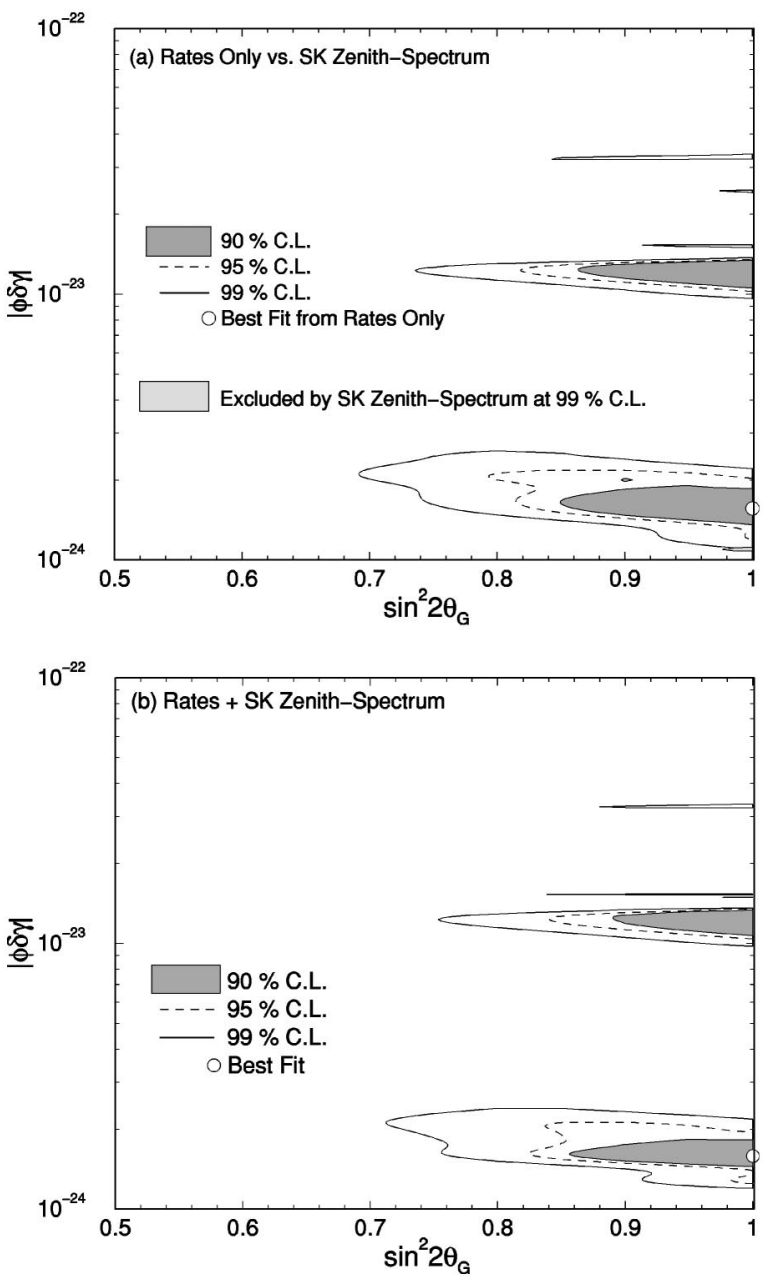

FIG. 5. Same as Fig. 1 but for the VEP solution in the $\sin ^{2} 2 \theta_{G}$ $-|\phi \delta \gamma|$ plane. Notice in the upper panel the very small exclusion region at $99 \%$ C.L. from the SK zenith-spectrum information appears at $\sin ^{2} 2 \theta_{G} \sim 1$ and $|\phi \delta \gamma| \sim 10^{-24}$.

solar neutrino observations alone cannot yet conclude if neutrinos have nonvanishing mass or magnetic moment. Furthermore, no stringent limit on the existence of NSNI nor on VEP can be currently set by the solar neutrino data.

The ultimate goal of the solar neutrino observations is, of course, to perform a direct experimental identification of the solution. For this purpose, we will have to wait for the upcoming solar neutrino experiments, which hopefully will provide a lot of new information, to reveal the true nature of the flavor conversion mechanism which is behind the SNP. For instance, low energy solar neutrino experiments, such as Borexino [53], can possibly discriminate among the solutions considered in this work, as can be seen in Fig. 7 of Ref. [54].

In the near future a new reactor experiment, KamLAND [55], may measure $\nu_{e}$ disappearance. For all mechanisms studied here, with the exception of LMA, we expect no significant disappearance in KamLAND because the baseline is too short to developed oscillation. Then a positive evidence for neutrino oscillation in KamLAND can establish the LMA solution. For the negative evidence no conclusion can be 
drawn to favor a particular mechanism studied in this work. A discussion about the consequences of the KamLAND experiment on the mechanism of the neutrino mass and the mixing hypothesis can be found in Ref. [56].

We summarize our conclusions.

(1) The present solar neutrino data only by themselves cannot discard, a priori, any of the solutions discussed here: (a) neutrino mass and mixing, (b) nonvanishing neutrino magnetic moment, (c) the existence of nonstandard flavor changing and nonuniversal neutrino interactions, and (d) the tiny violation of the equivalence principle. We refer to Tables III and IV for comparison. All solutions have a confidence level over $60 \%$ providing a very good fit to the solar neutrino data.

(2) A very robust statement is that LMA is the best preferred solution for mass-induced oscillation scenario, whereas the SMA is the worst one.

(3) Future experiments could test these different scenarios and possibly discard some of them.

\section{ACKNOWLEDGMENTS}

This work was supported by Fundação de do Estado de São Paulo (FAPESP) and by Conselho Nacional de Ciência e Tecnologia (CNPq).
[1] Homestake Collaboration, K. Lande et al., Astrophys. J. 496, 505 (1998).

[2] Kamiokande Collaboration, Y. Fukuda et al., Phys. Rev. Lett. 77, 1683 (1996).

[3] SAGE Collaboration, D. N. Abdurashitov et al., Nucl. Phys. B (Proc. Suppl.) 91, 36 (2001); latest results from SAGE homepage: http://EWIServer.npl.washington.edu/SAGE/

[4] GALLEX Collaboration, W. Hampel et al., Phys. Lett. B 447, 127 (1999).

[5] GNO Collaboration, M. Altmann et al., Phys. Lett. B 490, 16 (2000); C. Cattadori on behalf of GNO Collaboration, talk presented at TAUP2001, Laboratori Nazionali del Gran Sasso, Assergi, Italy, 2001.

[6] Super-Kamiokande Collaboration, Y. Fukuda et al., Phys. Rev. Lett. 82, 1810 (1999); 86, 5651 (2001).

[7] J. N. Bahcall, Neutrino Astrophysics (Cambridge University Press, Cambridge, England, 1989).

[8] J. N. Bahcall, S. Basu and M. H. Pinsonneault, Astrophys. J. 555, 990 (2001) and references therein; see also J.N. Bahcall's home page, http://www.sns.ias.edu/ $\sim$ jnb

[9] J. N. Bahcall, M. C. Gonzalez-Garcia, and C. Peña-Garay, hep-ph/0111150.

[10] SNO Collaboration, Q. R. Ahmad et al., Phys. Rev. Lett. 87, 071301 (2001).

[11] G. L. Fogli, E. Lisi, D. Montanino, and A. Palazzo, Phys. Rev. D 64, 093007 (2001); V. Barger, D. Marfatia, and K. Whisnant, Phys. Rev. Lett. 88, 011302 (2002); C. Giunti, Phys. Rev. D 65, 033006 (2002); V. Berezinsky, hep-ph/0108166.

[12] J. N. Bahcall, Phys. Rev. C 65, 015802 (2002).

[13] For a detailed list of the references on various conversion mechanisms, see Solar Neutrinos: The First Thirty Years, edited by R. Davis, Jr. et al., Frontiers in Physics Vol. 92 (Addison-Wesley, Reading, MA, 1994).

[14] Z. Maki, M. Nakagawa, and S. Sakata, Prog. Theor. Phys. 28, 870 (1962).

[15] V. N. Gribov and B. M. Pontecorvo, Phys. Lett. 28B, 493 (1969).

[16] L. Wolfenstein, Phys. Rev. D 17, 2369 (1978).

[17] S. P. Mikheyev and A. Yu. Smirnov, Sov. J. Nucl. Phys. 42, 913 (1985); Nuovo Cimento Soc. Ital. Fis., C 9, 17 (1986).

[18] D. O. Caldwell and R. N. Mohapatra, Phys. Rev. D 48, 3259 (1993); J. T. Peltoniemi, D. Tommasini, and J. W. F. Valle, Phys. Lett. B 298, 383 (1993); J. T. Peltoniemi and J. W. F.
Valle, Nucl. Phys. B406, 409 (1993); G. M. Fuller, J. R. Primack, and Y.-Z. Qian, Phys. Rev. D 52, 1288 (1995); J. J. Gomez-Cadenas and M. C. Gonzalez-Garcia, Z. Phys. C 71, 443 (1996); N. Okada and O. Yasuda, Int. J. Mod. Phys. A 12, 3669 (1997); G. Dvali and Y. Nir, J. High Energy Phys. 10, 014 (1998) and references therein.

[19] J. N. Bahcall, P. I. Krastev, and A. Yu. Smirnov, Phys. Rev. D 58, 096016 (1998); 60, 093001 (1999); M. C. Gonzalez-Garcia et al., Nucl. Phys. B573, 3 (2000); G. L. Fogli et al., Phys. Rev. D 62, 013002 (2000).

[20] A. M. Gago, H. Nunokawa, and R. Zukanovich Funchal, Phys. Rev. D 63, 013005 (2001); 64, 119902(E) (2001).

[21] The second reference of [19].

[22] J. N. Bahcall, M. C. Gonzalez-Garcia, and C. Peña-Garay, J. High Energy Phys. 08, 014 (2001); A. Bandyopadhyay et al., Phys. Lett. B 519, 83 (2001); P. I. Krastev and A. Yu. Smirnov, Phys. Rev. D (to be published), hep-ph/0108177; see also Ref. [12]; P. Aliani et al., hep-ph/0111418; see also Ref. [12].

[23] J. Schechter and J. W. F. Valle, Phys. Rev. D 24, 1883 (1981); 25, 283 (1982).

[24] C. S. Lim and W. J. Marciano, Phys. Rev. D 37, 1368 (1988); E. Kh. Akhmedov, Sov. J. Nucl. Phys. 48, 382 (1988); Phys. Lett. B 213, 64 (1988).

[25] For reviews, see for, e.g., J. Pulido, Phys. Rep. 211, 167 (1992); E. Kh. Akhmedov, in 4th International Solar Neutrino Conference, edited by W. Hampel (Neumann Druck, Heidelberg, Germany, 1997); hep-ph/9705451 and references therein.

[26] E. Kh. Akhmedov, A. Lanza, and S. T. Petcov, Phys. Lett. B 303, 85 (1993); P. I. Krastev, ibid. 303, 75 (1993); C. S. Lim and H. Nunokawa, Astropart. Phys. 4, 63 (1995); J. Pulido, Phys. Rev. D 57, 7108 (1998); B. C. Chauhan, U. C. Pandey, and S. Dev, Mod. Phys. Lett. A 13, 1163 (1998); M. M. Guzzo, N. Reggiani, and P. H. Sakanaka, Phys. Lett. B 357, 602 (1995); M. M. Guzzo, N. Reggiani, and J. H. Colonia, Phys. Rev. D 56, 588 (1997); J. Pulido and E. Kh. Akhmedov, Astropart. Phys. 13, 227 (2000); O. G. Miranda et al., Nucl. Phys. B595, 360 (2001).

[27] M. M. Guzzo and H. Nunokawa, Astropart. Phys. 12, 87 (1999).

[28] O. G. Miranda et al., Phys. Lett. B 521, 299 (2001).

[29] J. Pulido and E. Kh. Akhmedov, in Ref. [26].

[30] H. M. Antia, S. M. Chitre and M. J. Thompson, astro-ph/0005587. 
[31] M. M. Guzzo, A. Masiero, and S. T. Petcov, Phys. Lett. B 260, 154 (1991).

[32] J. W. F. Valle, Phys. Lett. B 199, 432 (1987); E. Roulet, Phys. Rev. D 44, 935 (1991); V. Barger, R. J. N. Phillips, and K. Whisnant, ibid. 44, 1629 (1991); S. Degl'Innocenti and B. Ricci, Mod. Phys. Lett. A 8, 471 (1993); G. L. Fogli and E. Lisi, Astropart. Phys. 2, 91 (1994); P. I. Krastev and J. N. Bahcall, hep-ph/9703267; S. Bergmann, Nucl. Phys. B515, 363 (1998).

[33] S. Bergmann et al., Phys. Rev. D 62, 073001 (2000); M. M. Guzzo, P. C. de Holanda, and H. Nunokawa, Nucl. Phys. B (Proc. Suppl.) 100, 62 (2001).

[34] M. M. Guzzo et al., Phys. Rev. D 64, 097301 (2001).

[35] M. Gasperini, Phys. Rev. D 38, 2635 (1988); 39, 3606 (1989).

[36] A. Halprin and C. N. Leung, Phys. Rev. Lett. 67, 1833 (1991).

[37] A. M. Gago, H. Nunokawa, and R. Zukanovich Funchal, Phys. Rev. Lett. 84, 4035 (2000); Nucl. Phys. B (Proc. Suppl.) 100, 68 (2001).

[38] I. R. Kenyon, Phys. Lett. B 237, 274 (1990).

[39] J. Pantaleone, A. Halprin, and C. N. Leung, Phys. Rev. D 47, R4199 (1993); J. N. Bahcall, P. I. Krastev, and C. N. Leung, ibid. 52, 1770 (1995); S. W. Mansour and T. K. Kuo, ibid. 60, 097301 (1999).

[40] J. Pantaleone, T. K. Kuo, and S. W. Mansour, Phys. Rev. D 61, 033011 (2000).

[41] CCFR Collaboration, A. Romosan et al., Phys. Rev. Lett. 78, 2912 (1997).

[42] A. Raychaudhuri and A. Sil, Phys. Rev. D (to be published), hep-ph/0107022.

[43] G. L. Fogli and E. Lisi, Astropart. Phys. 3, 185 (1995).

[44] P. C. de Holanda, Ph.D. thesis, IFGW/UNICAMP, 2001.
[45] A. R. Junghans et al., Phys. Rev. Lett. 88, 041101 (2002).

[46] S. Nakamura et al., Phys. Rev. C 63, 034617 (2001).

[47] Super-Kamiokande Collaboration, Y. Fukuda et al., Phys. Rev. Lett. 86, 5656 (2001).

[48] C. E. Ortiz et al., Phys. Rev. Lett. 85, 2909 (2000).

[49] See, e.g., M. C. Gonzalez-Garcia et al., Phys. Rev. D 58, 033004 (1998); Nucl. Phys. B543, 3 (1999); N. Fornengo, M. C. Gonzalez-Garcia, and J. W. F. Valle, ibid. B580, 58 (2000).

[50] LSND Collaboration, C. Athanassopoulos et al., Phys. Rev. Lett. 77, 3082 (1996); 81, 1774 (1998).

[51] P. Lipari and M. Lusignoli, Phys. Rev. D 60, 013003 (1999); G. L. Fogli et al., ibid. 60, 053006 (1999); S. Bergmann and Y. Grossman, ibid. 59, 093005 (1999); M. M. Guzzo et al., Nucl. Phys. B (Proc. Suppl.) 87, 201 (2000); N. Fornengo et al., Phys. Rev. D 65, 013010 (2002).

[52] After this work was submitted it was found by M. M. Guzzo et al., hep-ph/0112310, that it is possible to reconcile the solution by nonstandard flavor-changing and nonuniversal neutrino interactions to the SNP with the standard solution to the atmospheric neutrino anomaly.

[53] BOREXINO Collaboration, G. Ranucci et al., Nucl. Phys. B (Proc. Suppl.) 91, 58 (2001).

[54] H. Nunokawa, Proceedings of the conference Low Energy Solar Neutrino Detection, University of Tokyo, Japan, edited by Y. Suzuki, M. Nakahata, and S. Moriyama, hep-ph/0105027.

[55] KamLAND Collaboration, A. Piepke et al., Nucl. Phys. B (Proc. Suppl.) 91, 99 (2001).

[56] V. D. Barger, D. Marfatia, and B. P. Wood, Phys. Lett. B 498, 53 (2001); H. Murayama and A. Pierce, Phys. Rev. D 65, 013012 (2002); A. de Gouvea and C. Peña-Garay, ibid. 64, 113011 (2001); A. Strumia and F. Vissani, J. High Energy Phys. 11, 048 (2001). 